\title{
Calculation of Anharmonic Coupling Constants between Phonon Modes in GaAs
}

\author{
B. Kolad, A. Musią and P. Machnikowski \\ Institute of Physics, Wrocław University of Technology \\ Wybrzeże Wyspiańskiego 27, 50-370 Wrocław, Poland
}

\begin{abstract}
We derive third-order anharmonic coupling constants between various phonon modes in a zinc-blende crystal of a binary compound using a valence force field model extended by adding Coulomb interactions between polarizable ions. We find and compare the anharmonic coupling between the zone-center LO phonon and other phonon modes along principal directions of a GaAs crystal.
\end{abstract}

PACS numbers: 63.20.kg, 63.20.dh

\section{Introduction}

Anharmonic coupling between phonon modes of a crystal determine many of its properties, thermal conductivity being a standard example. Although anharmonicity-related effects observed in bulk crystals (decay of the zone-center longitudinal optical (LO) phonon or acoustic wave attenuation) rely only on a small subset of anharmonic couplings, the full set of such constants is needed for a complete description of phonon-induced dephasing and relaxation of electrons and excitons in quantum dots [1-4]. The reason for this is that breaking of the translational symmetry and appearance of a quantized spectrum completely changes the energy-momentum conservation conditions and opens decoherence (relaxation and dephasing) paths which are not allowed in a bulk crystal. So far, only the anharmonic couplings between acoustic branches have been completely described [5], while the only known coupling involving optical phonons is that between the LO phonon and two longitudinal acoustic (LA) phonons, which seems to be responsible for the decay of the zone-center LO phonon in a bulk crystal [6] and is therefore accessible experimentally [7].

In this paper, we derive third-order anharmonic coupling constants between various phonon modes in a zinc-blende crystal. We use a model combining the 
forces resulting from bond distortions (valence force field approach) with long-range Coulomb interactions between ions, which are, in addition, assumed to be polarizable. Thus, our model generalizes the Keating-Martin model [8, 9] by including polarizability of the ions. Couplings involving the zone-center LO phonon are of particular interest because of their importance for the carrier-phonon kinetics in confined systems, which is due to a strong polaron effect.

\section{The model and the method}

We take the potential energy of the lattice in the form $U=U^{(2)}+U^{(3)}$, where

$$
\begin{aligned}
U^{(2)} & =\frac{1}{2} \sum_{l}\left[\frac{\alpha}{4 a_{0}^{2}} \sum_{i}\left(x_{l i}^{2}-3 a_{0}^{2}\right)^{2}\right. \\
& \left.+\frac{\beta_{l}}{2 a_{0}^{2}} \sum_{i, j>i}\left(\boldsymbol{x}_{l i} \cdot \boldsymbol{x}_{l j}+a_{0}^{2}\right)^{2}-\sum_{i} V_{l i}^{(\mathrm{C})}\right]
\end{aligned}
$$

and

$$
\begin{aligned}
U^{(3)} & =\frac{1}{3} \sum_{l}\left[\frac{\gamma}{2 a_{0}^{3}} \sum_{i}\left(x_{l i}^{2}-3 a_{0}^{2}\right)^{3}\right. \\
& \left.+\frac{\delta}{a_{0}^{3}} \sum_{i, j<i}\left(\boldsymbol{x}_{l i} \cdot \boldsymbol{x}_{l j}+a^{2}\right)^{3}+\frac{\epsilon}{2 a_{0}^{3}} \sum_{i \neq j}\left(x_{l i}^{2}-3 a^{2}\right)\left(\boldsymbol{x}_{l i} \cdot \boldsymbol{x}_{l j}+a_{0}^{2}\right)^{2}\right] .
\end{aligned}
$$

Here $\boldsymbol{x}_{l i}=\boldsymbol{x}_{l}-\boldsymbol{x}_{i}$, where $\boldsymbol{x}_{i}$ is the position of the $i$-th ion, $a_{0}$ is the equilibrium length of the bond and $\alpha, \beta_{l}, \gamma, \delta, \epsilon$ are force constants.

The component $U^{(2)}$ contains terms of 2 nd and higher orders in the ionic displacements, while the leading terms of $U^{(3)}$ are of the 3rd order. In Eq. (1), the first and second terms account for central bond-stretching forces and noncentral bond bending forces, respectively. These terms are essentially taken from the Keating model [8], but we allow the two ions to have different bond bending parameters $\beta_{l}$. The third term is the Coulomb interaction energy between ions, including the polarizability of the ions in the local electric field. This term is parametrized by three parameters: the effective ion charge and the polarizabilities of the two ions. Equation (2) is again taken from the work by Keating [10].

The calculations proceed in two steps. First, we derive the dynamical matrix from the potential energy $U^{(2)}$, find the phonon frequencies and polarizations for an arbitrary wave vector, and find the parameters of the model by a least squares fit to the measured dispersion curves [11]. Next, we express the third-order terms derived from $U^{(2)}$ and $U^{(3)}$ in terms of these numerically found phonon modes which directly yields anharmonic couplings between them. In this procedure, we use anharmonic force constants $\gamma, \delta, \epsilon$ taken from literature [12], where they are derived from the third order macroscopic elastic constants. These anharmonic 
constants already include third-order terms arising from the Coulomb interactions between nearest and next-nearest neighbors, while the interactions between more remote ions should be considerably weaker. Therefore, we do not include the latter in our calculations.

The dynamical matrix of the lattice is

$$
\begin{aligned}
& D_{k \alpha ; k^{\prime} \alpha^{\prime}}(\boldsymbol{q})=\frac{1}{\sqrt{m_{k} m_{k^{\prime}}}} \sum_{n^{\prime}} \frac{\partial^{2} U^{(2)}}{\partial x_{n k \alpha} \partial x_{n^{\prime} k^{\prime} \alpha^{\prime}}} \mathrm{e}^{\mathrm{i} \boldsymbol{q} \cdot\left(\boldsymbol{R}_{n k}-\boldsymbol{R}_{n^{\prime} k^{\prime}}\right)} \\
& =D_{k \alpha ; k^{\prime} \alpha^{\prime}}^{(\mathrm{VF})}(\boldsymbol{q})+D_{k \alpha ; k^{\prime} \alpha^{\prime}}^{(\mathrm{C})}(\boldsymbol{q}),
\end{aligned}
$$

where $n, n^{\prime}$ run through all the primitive cells, $k, k^{\prime}=1,2$ number ions in a single cell, Greek letters denote Cartesian coordinates of vectors, $\boldsymbol{R}_{n k}$ are the equilibrium positions of the ions, and we split the dynamical matrix into the valence force and Coulomb parts, corresponding to the first two and the third terms in Eq. (1).

The summations over $i$ and $j$ in Eq. (1) involve only a small number of nearest and next-nearest neighbors, hence $D^{(\mathrm{VF})}$ can be found from $U^{(2)}$ in a straightforward manner. Calculation of the forces resulting from the Coulomb term involves summation over the whole infinite crystal. This is done using the Ewald method [13], in which the summation is done partly in the real space and partly in the reciprocal space, each contributions being regular and quickly convergent. As a result, one gets the Coulomb part in the implicit form [13]:

$$
\begin{aligned}
& \sum_{k^{\prime} \beta} D_{k \alpha ; k^{\prime} \beta}^{(\mathrm{C})}(\boldsymbol{q}) u_{k^{\prime} \beta}(\boldsymbol{q})=-\sum_{k^{\prime} \beta} \frac{1}{\sqrt{m_{k} m_{k^{\prime}}}} \\
& \quad \times\left[e_{k} e_{k^{\prime}} B_{k \alpha ; k^{\prime} \beta}(\boldsymbol{q})-\delta_{k k^{\prime}} \sum_{k^{\prime \prime}} e_{k} e_{k^{\prime \prime}} B_{k \alpha ; k^{\prime \prime} \beta}(0)\right] u_{k^{\prime} \beta}(\boldsymbol{q})-\frac{1}{\sqrt{m_{k}}} \\
& \quad \times \sum_{k^{\prime} \beta}\left[e_{k} B_{k \alpha ; k^{\prime} \beta}(\boldsymbol{q})-\delta_{k k^{\prime}} \sum_{k^{\prime \prime}} e_{k^{\prime \prime}} B_{k \alpha ; k^{\prime \prime} \beta}(0)\right] p_{k^{\prime} \beta}(\boldsymbol{q})-\frac{1}{\sqrt{m_{k}}} E_{\alpha},
\end{aligned}
$$

where $\boldsymbol{u}_{k}(\boldsymbol{q})$ is the Fourier component of the displacement of the $k$-th ion,

$$
\begin{aligned}
B_{k \alpha} ; k^{\prime} \beta & (\boldsymbol{q})=\frac{4 \pi}{v}\left(\frac{q_{\alpha} q_{\beta}}{q^{2}}\right)\left(1-\mathrm{e}^{-\pi^{2} q^{2} / Q_{0}^{2}}\right) \\
+ & Q_{0}^{3} \sum_{\boldsymbol{R}} H_{\alpha \beta}\left[Q_{0}\left(\boldsymbol{R}+\boldsymbol{\xi}_{k k^{\prime}}\right)\right] \mathrm{e}^{2 \pi \mathrm{i} \boldsymbol{q} \cdot\left(\boldsymbol{R}+\boldsymbol{\xi}_{k k^{\prime}}\right)} \\
& -\frac{4 \pi^{3}}{Q_{0}^{2} v} \sum_{\boldsymbol{Q}}^{\prime}\left(q_{\alpha}+Q_{\alpha}\right)\left(q_{\beta}+Q_{\beta}\right) G\left(\frac{\pi^{2}|\boldsymbol{q}+\boldsymbol{Q}|^{2}}{Q_{0}^{2}}\right) \mathrm{e}^{2 \pi \mathrm{i} \boldsymbol{Q} \cdot \boldsymbol{\xi}_{k k^{\prime}}},
\end{aligned}
$$

$v$ is the volume of a primitive cell, the first sum runs through the lattice vectors $\boldsymbol{R}$, the second sum runs through the vectors of the reciprocal lattice $\boldsymbol{Q}$, excluding $\boldsymbol{Q}=0, \boldsymbol{\xi}_{k k^{\prime}}=\boldsymbol{\xi}_{k}-\boldsymbol{\xi}_{k^{\prime}}$, where $\boldsymbol{\xi}_{k}$ is the equilibrium position of the ion $k$ in a 
primitive cell with respect to the cell origin, $Q_{0}$ is the parameter of the Ewald method, $e_{k}$ is the charge of the $k$-th ion,

$$
H_{\alpha \beta}(\boldsymbol{x})=\frac{\partial^{2}}{\partial x_{\alpha} \partial x_{\beta}}\left(\frac{2}{\sqrt{\pi} x} \int_{x}^{\infty} \mathrm{e}^{-x^{2}} \mathrm{~d} x\right), \quad G(x)=\frac{\mathrm{e}^{-x}}{x},
$$

and in the term $\boldsymbol{R}=0, k=k^{\prime}$ the function $H_{\alpha \beta}(x)$ should be replaced by

$$
H_{\alpha \beta}^{(0)}(\boldsymbol{x})=-\frac{\partial^{2}}{\partial x_{\alpha} \partial x_{\beta}}\left(\frac{2}{\sqrt{\pi} x} \int_{0}^{x} \mathrm{e}^{-x^{2}} \mathrm{~d} x\right) .
$$

Furthermore, $\boldsymbol{E}(\boldsymbol{q})$ is the Fourier component of the macroscopic electric field, and $\boldsymbol{p}_{k}(\boldsymbol{q})$ is the Fourier component of the electric dipole moment of the ion $k$. The latter two quantities are related to the displacement amplitudes by the equations

$$
E_{\alpha}=-\sum_{k^{\prime} \beta} \frac{4 \pi}{v}\left(\frac{q_{\alpha} q_{\beta}}{q^{2}}\right)\left[p_{k^{\prime} \beta}(\boldsymbol{q})+\frac{e_{k^{\prime}}}{\sqrt{m_{k^{\prime}}}} u_{k^{\prime} \beta}(\boldsymbol{q})\right]
$$

and

$$
\begin{gathered}
p_{k \alpha}(\boldsymbol{q})=\alpha_{k}\left\{E_{\alpha}(\boldsymbol{q})+\sum_{k^{\prime} \beta}\left[e_{k^{\prime}} B_{k \alpha ; k^{\prime} \beta}(\boldsymbol{q})-\delta_{k k^{\prime}} \sum_{k^{\prime \prime}} e_{k^{\prime \prime}} B_{k \alpha ; k^{\prime \prime} \beta}(0)\right]\right. \\
\left.\times \frac{1}{\sqrt{m_{k^{\prime}}}} u_{k^{\prime} \beta}(\boldsymbol{q})+\sum_{k^{\prime} \beta} B_{k \alpha ; k^{\prime} \beta}(\boldsymbol{q}) p_{k^{\prime} \beta}(\boldsymbol{q})\right\},
\end{gathered}
$$

where $\alpha_{k}$ is the atomic polarizability of the $k$-th ion.

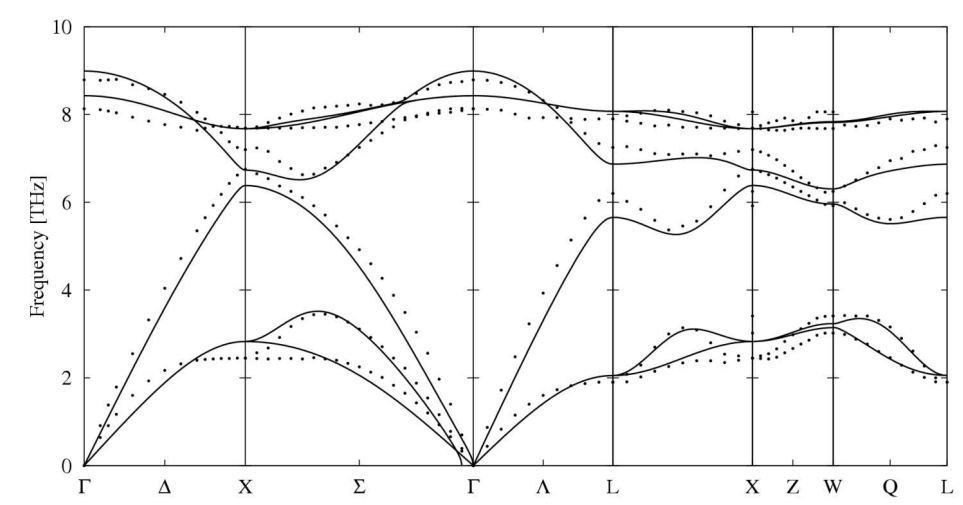

Fig. 1. The phonon dispersion curves obtained from our model with fitted parameters (lines), compared to the experimental data [11] (points).

By varying the valence force parameters $\alpha, \beta_{l}$ as well as the effective charges $e_{k}$ (obviously $e_{1}=-e_{2}$ ) and polarizabilities $\alpha_{k}$, the best least squares fit of the dispersion curves obtained by diagonalizing the dynamical matrix to the measured values for GaAs [11] is found. The resulting curves are shown in Fig. 1. The phonon dispersion curves obtained within our relatively simple six-parameter model repro- 
duce the experimental data with a reasonable accuracy, with discrepancies mostly at the edges of the Brillouin zone.

The lowest order term beyond the harmonic approximation has the general form

$$
H_{3}=\frac{1}{3 !} \sum_{k k^{\prime} k^{\prime \prime}} \sum_{n n^{\prime} n^{\prime \prime}} \sum_{\alpha \alpha^{\prime} \alpha^{\prime \prime}} \frac{\partial^{3} U^{(3)}}{\partial x_{n k \alpha} \partial x_{n^{\prime} k^{\prime} \alpha^{\prime}} \partial x_{n^{\prime \prime} k^{\prime \prime} \alpha^{\prime \prime}}} s_{n k \alpha} s_{n^{\prime} k^{\prime} \alpha^{\prime}} s_{n^{\prime \prime} k^{\prime \prime} \alpha^{\prime \prime}},
$$

where

$$
s_{n k \alpha}=\frac{1}{\sqrt{N M_{\alpha}}} \sum_{j \boldsymbol{q}}\left(\frac{\hbar}{2 \omega_{\boldsymbol{q} j}}\right)^{1 / 2} e_{\alpha i}^{j}(\boldsymbol{q}) \exp \left(\mathrm{i} \boldsymbol{q} \cdot \boldsymbol{R}_{\boldsymbol{n}, k}\right)\left(a_{-\boldsymbol{q} j}^{\dagger}+a_{\boldsymbol{q} j}\right)
$$

are the displacements of the ions from their equilibrium positions. Here $j, j^{\prime}, j^{\prime \prime}$ denote phonon branches. We find the third derivative appearing in Eq. (3) explicitly from Eq. (2), substitute the ion displacements from Eq. (4), and write $H_{3}$ in the form

$$
\begin{aligned}
H_{3}= & \frac{\hbar^{1 / 2}}{3 ! \sqrt{N}} \sum_{\boldsymbol{q} \boldsymbol{q}^{\prime} \boldsymbol{q}^{\prime \prime}} \sum_{j j^{\prime} j^{\prime \prime}} w_{\boldsymbol{q} \boldsymbol{q}^{\prime} \boldsymbol{q}^{\prime \prime}}^{j j^{\prime \prime}} \delta_{\boldsymbol{q}+\boldsymbol{q}^{\prime}+\boldsymbol{q}^{\prime \prime}, \boldsymbol{K}}\left(a_{-\boldsymbol{q} j}^{\dagger}+a_{\boldsymbol{q} j}\right)\left(a_{-\boldsymbol{q}^{\prime} j^{\prime}}^{\dagger}+a_{\boldsymbol{q}^{\prime} j^{\prime}}\right) \\
& \times\left(a_{-\boldsymbol{q}^{\prime \prime} j}^{\dagger}+a_{\boldsymbol{q}^{\prime \prime} j^{\prime \prime}}\right),
\end{aligned}
$$

where $w_{\boldsymbol{q}^{\prime} \boldsymbol{q}^{\prime \prime}}^{j j^{\prime} j^{\prime \prime}}$ are the anharmonic coupling constants of interest.

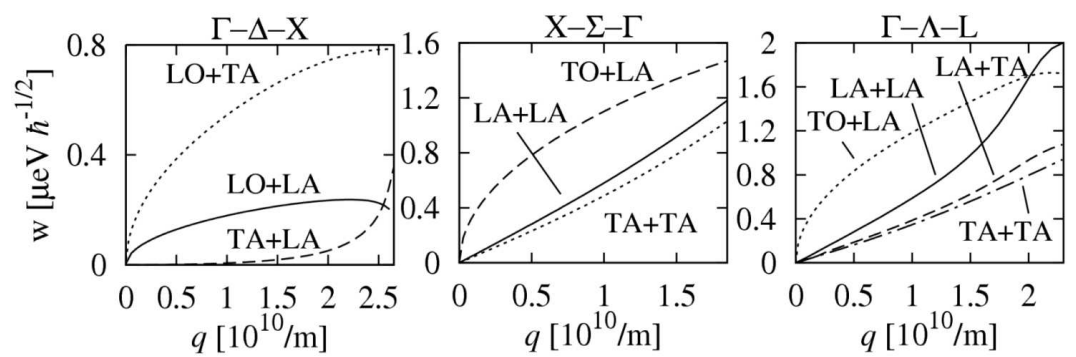

Fig. 2. Third order anharmonic coupling coefficients for the coupling between a zone-center LO phonon and two phonons belonging to the branches as indicated, with momenta $\boldsymbol{q}$ and $-\boldsymbol{q}$, as a function of $q$, along selected directions in the Brillouin zone, as shown.

The anharmonic constants $w_{q q^{\prime} q^{\prime \prime}}^{j j^{\prime \prime}}$ found in this way are shown in Fig. 2. For each of the principal crystallographic directions originating at the $\Gamma$ point of the Brillouin zone, we plot the constants $w_{\boldsymbol{q}=0, \boldsymbol{q}^{\prime},-\boldsymbol{q}^{\prime \prime}}^{\mathrm{LO}}$ for the branches that yield the strongest anharmonicity.

\section{Conclusions}

We have developed procedures for calculating anharmonic couplings between arbitrary phonon modes in a zinc-blende crystal. This allowed us to compute 
anharmonic couplings involving long-wavelength LO phonons, which are essential, e.g., for the description of relaxation and dephasing processes in quantum dots. It turns out that anharmonic couplings for individual modes are strongly directiondependent. Our results show also that couplings involving an optical and acoustic branch tend to be stronger than those involving two acoustic branches.

\section{References}

[1] L. Jacak, P. Machnikowski, J. Krasnyj, P. Zoller, Eur. Phys. J. D 22, 319 (2003).

[2] P. Machnikowski, Phys. Rev. Lett. 96, 140405 (2006).

[3] P. Machnikowski, Phys. Status Solidi B 243, 2247 (2006).

[4] E.A. Zibik, L.R. Wilson, R.P. Green, G. Bastard, R. Ferreira, P.J. Phillips, D.A. Carder, J.-P.R. Wells, J.W. Cockburn, M.S. Skolnick, M.J. Steer, M. Hopkinson, Phys. Rev. B 70, 161305 (2004).

[5] S. Tamura, Phys. Rev. B 31, 2574(R) (1985).

[6] S. Barman, G.P. Srivastava, Appl. Phys. Lett. 81, 3395 (2002).

[7] F. Vallée, F. Bogani, Phys. Rev. B 43, 12049 (1991).

[8] P.N. Keating, Phys. Rep. 145, 637 (1966).

[9] R.M. Martin, Phys. Rev. B 1, 4005 (1970).

[10] P.N. Keating, Phys. Rep. 149, 674 (1966).

[11] D. Strauch, B. Dorner, J. Phys., Condens. Matter 2, 1457 (1990).

[12] R. Singh, G. Verma, J. Appl. Phys. 39, 4032 (1968).

[13] M. Born, K. Huang, Dynamical Theory of Crystal Lattices, Oxford University Press, Oxford 1954. 